\title{
A DEGREE THEORY FOR VARIATIONAL INEQUALITIES WITH SUMS OF MAXIMAL MONOTONE AND $\left(\mathbf{S}_{+}\right)$OPERATORS
}

\author{
IN-SoOK Kim - MarTin VÄTH
}

\begin{abstract}
We develop a degree theory for variational inequalities which contain multivalued $\left(\mathrm{S}_{+}\right)$-perturbations of maximal monotone operators. The multivalued operators need not necessarily be convex-valued. The result is simultaneously an extension of a degree theory for variational inequalities (developed by Benedetti, Obukhovskir and Zecca) and of the Skrypnik-Browder degree and extensions thereof.
\end{abstract}

\section{Introduction}

Throughout, let $X$ be a real Banach space with dual space $X^{*}$, the usual pairing being denoted by $\langle\cdot, \cdot\rangle: X \times X^{*} \rightarrow \mathbb{R}$, i.e. $\left\langle x, x^{*}\right\rangle:=x^{*}(x)$. Let $K \subseteq X$ be closed and convex. Given $\Phi: X \rightarrow 2^{X^{*}}$ and $M \subseteq X$, we denote by $\operatorname{ineq}_{M}(\Phi, K)$ the set of all $x \in M$ which satisfy the following variational inequality for some $y \in \Phi(x):$

$$
x \in K, \quad\langle\xi-x, y\rangle \geq 0 \quad \text { for all } \xi \in K
$$

2010 Mathematics Subject Classification. Primary: 47H11; Secondary: 47H04, 47H05, 47H14, 47J20, 47J22.

Key words and phrases. Degree theory; maximal monotone operator; operator of type $\left(\mathrm{S}_{+}\right)$; multivalued map; variational inequality; Browder-Skrypnik degree.

The paper was written in the framework of a DFG project (Az. VA 206/2-1) and of the Basic Science Research Program through the National Research Foundation of Korea (NRF) funded by the Ministry of Education, Science and Technology (NRF-2012-0008345). Financial support by the DFG and the NRF is gratefully acknowledged. 
Note that in case $K=X$, this means $\operatorname{ineq}_{M}(\Phi, X)=\{x \in M: 0 \in \Phi(x)\}$.

We are interested in a topological degree which for bounded open $U \subseteq X$ "counts" the number of elements of $\operatorname{ineq}_{U}(\Phi, K)$ in a certain sense which is homotopically invariant. For the case $K=X$ such a degree theory was developed independently by Skrypnik [12] and Browder [7] if $\Phi$ is a single-valued map of so-called type $\left(\mathrm{S}_{+}\right)$. For $K=X$ and $\Phi=T+S$ with a maximal monotone map $T$ satisfying $0 \in T(0)$ and a single-valued map $S$ of type $\left(\mathrm{S}_{+}\right)$this was generalized in [8], the condition $0 \in T(0)$ was dropped in [11]. All these results were generalized to multivalued maps $S$ with convex values [15] and, moreover, to the case that $S$ is only pseudomonotone. The most general result of such a type which we know currently is [1] where many historical remarks can also be found.

On the other hand, the case $K \neq X$ is to our knowledge only studied in [2] (see [3] for the finite-dimensional case), even for multivalued mappings $\Phi=S$ which are of type $\left(\mathrm{S}_{+}\right)$and which need not necessarily have convex values $S(x)$ but e.g. can be written in the form $\varphi \circ \Phi$ where $\varphi$ is single-valued and $\Phi$ belongs to a certain class of approximable mappings, e.g. $\Phi$ is upper semicontinuous with $\Phi(x)$ being an $R_{\delta}$-set for every $x$. Recall that an $R_{\delta}$-set is the intersection of a decreasing sequence of compact contractible metrizable spaces.

It is the aim of our paper to obtain such a degree even for maps of the form $\Phi=T+S$ where $S$ is as above and $T$ is maximal monotone. To our knowledge, the results are new even in case $K=X$, since we do not require that $S$ assumes convex values and since we do not require that $T=0$. It is somewhat amusing that $T(x)$ is convex (since $T$ is maximal monotone) while even in case $\varphi=\mathrm{id}$ the values $S(x)$ are topologically "trivial" in a sense, but not convex in general, so that the values of the (Minkowski) sum $T(x)+S(x)$ are typically far from being topologically trivial.

However, this is only a minor advantage since in most applications $S$ will also have convex values: The crucial advantage of the degree of our paper over that from e.g. [1] is that we treat variational inequalities which seem to be really new: The degree theory for inequalities from [2] cannot directly treat the case that the considered operators assume empty or unbounded values which is in many applications the case for maximal monotone operators $T$. Thus, as far as we know, for the case $K \neq X$ and with $T \neq 0$, our results are completely new.

It is probably possible to obtain a degree as in our paper also when $S$ is only pseudomonotone instead of class $\left(\mathrm{S}_{+}\right)$, analogously to [1]. However, the hypothesis concerning nondegeneracy on the boundary in [1] is already almost impossible to check, and for variational inequalities it becomes even more technical and artificial so that we do not strive for this further generalization in this paper. We note, however, that for $T=0$ the case of pseudomonotone $S$ does not 
cause this difficulty, and for this setting, the corresponding theory has already been developed satisfactorily in [2].

\section{Main results}

We use the notation $\Phi: X \multimap Y$ to denote a multivalued map with values in $Y$, that is, $\Phi(x)$ is for every $x \in X$ a (possibly empty) subset of $Y$. We put $D(\Phi):=\{x \in X: \Phi(x) \neq \emptyset\}$. Notationally, we do not distinguish further between single- and multivalued maps (which is a slight misuse in notation, of course). We put graph $\Phi:=\{(x, y): y \in \Phi(x)\}$, and denote by $\Phi^{-1}: Y \multimap X$ the map with $\operatorname{graph}\left(\Phi^{-1}\right)=\{(y, x):(x, y) \in \operatorname{graph} \Phi\}$, that is, $\Phi^{-1}(y):=\{x$ : $y \in \Phi(x)\}$. For a set $M \subseteq X$, we call as customary the set $\{\Phi(x): x \in M\}$ the values of $\Phi$ on $M$, and we denote the union of these values by $\Phi(M)$ and call it the image of $M$ under $\Phi$. If $\Phi: X \multimap Y$ and $\varphi: Y \rightarrow Z$ then $\varphi \circ \Phi: X \multimap Z$ is defined by $(\varphi \circ \Phi)(x)=\varphi(\Phi(x))$.

Throughout, let $X$ be a real separable reflexive Banach space with dual space $X^{*}$, and let $K \subseteq X$ be closed and convex. For $M, N \subseteq X^{*}$ we denote by $M+N:=\{u+v: u \in M, v \in N\}$ the Minkowski sum, and in this sense we also understand the sum of multivalued maps $T, S: X \multimap X^{*}$ as $(T+S)(x):=$ $T(x)+S(x)$.

A map $T: X \multimap X^{*}$ is called monotone if $\langle x-y, u-v\rangle \geq 0$ whenever $u \in$ $T(x), v \in T(y)$, and maximal monotone if its graph is maximal with respect to the inclusion order of the family of all graphs of monotone maps, that is, if for any $x \in X$ and $u \in X^{*},\langle x-y, u-v\rangle \geq 0$ whenever $v \in T(y)$ imply $u \in T(x)$.

Let $M \subseteq X$, we say that a family $S:[0,1] \times X \multimap X^{*}$ belongs to $\mathcal{S}^{+}([0,1] \times M)$ if the following holds:

(1) $S([0,1] \times(B \cap M))$ is bounded for every bounded $B \subseteq X$.

(2) For all sequences $t_{n} \in[0,1], x_{n} \in M, y_{n} \in S\left(t_{n}, x_{n}\right)$, the assumptions $t_{n} \rightarrow t, x_{n} \rightarrow x, y_{n} \rightarrow y$, and

$$
\limsup _{n \rightarrow \infty}\left\langle x_{n}, y_{n}\right\rangle \leq\langle x, y\rangle
$$

imply that $x_{n} \rightarrow x$ and $y \in S(t, x)$.

Similarly, we say that $S: X \multimap X$ belongs to $\mathcal{S}^{+}(M)$ if the constant family $S(t, x)=S(x)$ belongs to $\mathcal{S}^{+}([0,1] \times M)$.

REMARK 2.1. Assumption (2.1) can equivalently be replaced by

$$
\limsup _{n \rightarrow \infty}\left\langle x_{n}-x, y_{n}\right\rangle \leq 0 \text {. }
$$

Indeed, $\left\langle x_{n}, y_{n}\right\rangle-\langle x, y\rangle=\left\langle x_{n}-x, y_{n}\right\rangle-\left\langle x, y-y_{n}\right\rangle$ with the last summand tending to 0 . 
REMARK 2.2. In order to verify that $S \in \mathcal{S}^{+}([0,1] \times M)$, it suffices to show that $[0,1] \ni t_{n} \rightarrow t, M \ni x_{n} \rightarrow x, S\left(t_{n}, x_{n}\right) \ni y_{n} \rightarrow y$ and (2.1) or (2.2) imply that $y \in S(t, x)$ and that $x_{n_{k}} \rightarrow x$ for some subsequence $x_{n_{k}}$. Indeed, if each subsequence of $x_{n}$ contains a subsequence convergent to $x$ then also $x_{n} \rightarrow x$.

Some admissible perturbations of the class $\mathcal{S}^{+}(M)$ are described in the following result. We call $T: I \times X \multimap X^{*}$ a jointly monotone family if

$$
\langle x-y, u-v\rangle \geq 0 \quad \text { whenever } u \in T(I \times\{x\}), v \in T(I \times\{y\}) .
$$

We call $\Phi: X \multimap X^{*}$ sequentially demiclosed if graph $\Phi$ is sequentially closed in $X \times X^{*}$ when $X$ is equipped with the strong topology and $X^{*}$ is equipped with the weak (i.e. weak*) topology. Similarly, we call $\Phi:[0,1] \times X \multimap X^{*}$ sequentially demiclosed if graph $\Phi$ is sequentially closed in $[0,1] \times X \times X^{*}$.

In other words, $\Phi:[0,1] \times X \multimap X^{*}$ is sequentially demiclosed if it follows from $t_{n} \in[0,1], x_{n} \in X, t_{n} \rightarrow t, x_{n} \rightarrow x \in X, y_{n} \in \Phi\left(t_{n}, x_{n}\right)$, and $y_{n} \rightarrow y$ that $y \in \Phi(t, x)$.

Proposition 2.3. Let $S \in \mathcal{S}^{+}([0,1] \times M)$. Let $T:[0,1] \times X \multimap X^{*}$ be a jointly monotone family which assumes only nonempty values, and let $C:[0,1] \times$ $X \multimap X^{*}$ be such that for each bounded set $B \subseteq X$ the set $T([0,1] \times(B \cap M))$ is bounded and $C([0,1] \times(B \cap M))$ is relatively compact. Assume furthermore that $T$ and $C$ are sequentially demiclosed. Then $\Phi:=T+S+C \in \mathcal{S}^{+}([0,1] \times M)$. An analogous assertion holds for $S \in \mathcal{S}^{+}(M)$.

Proof. The boundedness hypothesis of $\Phi$ is trivially satisfied. Thus, let sequences $t_{n} \in[0,1], x_{n} \in M, u_{n} \in T\left(t_{n}, x_{n}\right), v_{n} \in S\left(t_{n}, x_{n}\right), w_{n} \in C\left(t_{n}, x_{n}\right)$ be given such that $t_{n} \rightarrow t, x_{n} \rightarrow x, y_{n}:=u_{n}+v_{n}+w_{n} \rightarrow y$, and such that (2.2) holds. Since $X$ is reflexive and $u_{n}, v_{n}, w_{n}$ are bounded or relatively compact, respectively, we can assume in view of Remark 2.2 that $u_{n} \rightarrow u, v_{n} \rightarrow v, w_{n} \rightarrow w$ with $y=u+v+w$. The monotonicity of $T$ implies for some $z \in T(t, x)$ that

$$
\begin{aligned}
\left\langle x_{n}-x, y_{n}\right\rangle & =\left\langle x_{n}-x, u_{n}\right\rangle+\left\langle x_{n}-x, v_{n}\right\rangle+\left\langle x_{n}-x, w_{n}\right\rangle \\
& \geq\left\langle x_{n}-x, z\right\rangle+\left\langle x_{n}-x, v_{n}\right\rangle+\left\langle x_{n}-x, w\right\rangle+\left\langle x_{n}-x, w_{n}-w\right\rangle .
\end{aligned}
$$

Since $x_{n} \rightarrow x$ and $w_{n} \rightarrow w$, we thus obtain from (2.2) that $\limsup _{n \rightarrow \infty}\left\langle x_{n}-x, v_{n}\right\rangle \leq 0$. From $S \in \mathcal{S}^{+}([0,1] \times M)$, we conclude that $x_{n} \rightarrow x$ and $v \in S(t, x)$. Since $\left(t_{n}, x_{n}\right) \rightarrow(t, x), u_{n} \rightarrow u$, and $w_{n} \rightarrow w$, and since $T$ and $C$ are sequentially demiclosed, we obtain that $u \in T(t, x)$ and $w \in C(t, x)$ and thus $y \in \Phi(t, x)$. Therefore, we have shown that $\Phi \in \mathcal{S}^{+}([0,1] \times M)$.

Recall that a multivalued map $\Phi: M \multimap Y$ in topological spaces is called upper semicontinuous at $x_{0} \in M$ if for each open set $V \subseteq Y$ containing $\Phi\left(x_{0}\right)$ there is a neighbourhood $U \subseteq M$ of $x_{0}$ with $\Phi(U) \subseteq V$. If $\Phi$ is upper semicontinuous at every point of $M$, we call $\Phi$ upper semicontinuous on $M$. 
REmark 2.4. If $M \subseteq X$ is closed and $\Phi: M \multimap Y$ is upper semicontinuous then we can extend $\Phi$ trivially to an upper semicontinuous map $\Phi: X \multimap Y$ by putting $\Phi(x)=\emptyset$ for $x \notin M$. For this reason, it will be no restriction for us to consider only maps $\Phi: X \multimap Y$.

If $Y$ is a Banach space, we call $\Phi: M \multimap Y$ upper demicontinuous if it is upper semicontinuous when we equip $Y$ with the weak topology.

Proposition 2.5. If $S: X \multimap X^{*}\left(\right.$ or $\left.S:[0,1] \times X \multimap X^{*}\right)$ is upper demicontinuous with weakly closed values then $S$ is sequentially demiclosed.

Proof. By e.g. [13, Corollary 2.117], $\operatorname{graph}(S)$ is closed in $X \times X^{*}$ (with the norm topology on $X$ and weak topology on $X^{*}$ ) and thus sequentially closed.

Corollary 2.6. If $S: X \multimap X^{*}\left(\right.$ or $\left.S:[0,1] \times X \multimap X^{*}\right)$ is upper semicontinuous or upper demicontinuous and has compact values then $S$ is sequentially demiclosed.

Proof. If $S$ is upper semicontinuous then it is upper demicontinuous. Moreover, compact sets are weakly compact and thus also weakly closed.

Recall that a nonempty compact subset $M$ of a topological space $\Gamma$ is called aspheric in $\Gamma$ if every open set $U \subseteq \Gamma$ with $M \subseteq U$ contains an open set $V \subseteq U$ with $M \subseteq V$ such that for every $n=1,2, \ldots$ every continuous map $f$ from the unit sphere $S^{n-1} \subseteq \mathbb{R}^{n}$ with $f\left(S^{n-1}\right) \subseteq V$ has a continuous extension $f$ to the closed unit ball $B^{n} \subseteq \mathbb{R}^{n}$ with $f\left(B^{n}\right) \subseteq U$. If $\Gamma$ is an ANR, i.e. homeomorphic to a retract of a (relatively) open subset of a finite union of closed convex subsets of a normed space, then every $R_{\delta}$ subset of $\Gamma$ is aspheric in $\Gamma$.

We say $\Phi \in \mathcal{J}(M, \Gamma)$ if $\Phi: M \multimap \Gamma$ is upper semicontinuous and if $\Phi(x)$ is aspheric in $\Gamma$ for every $x \in M$. Similarly, we say $\Phi \in \mathcal{K}(M, \Gamma)$ if $\Gamma$ is an ANR subset of a normed space, and $\Phi$ is upper semicontinuous with $\Phi(x)$ being nonempty, convex, and compact for every $x \in M$. Since every nonempty convex compact set is an $R_{\delta}$, we have clearly $\mathcal{K}(M, \Gamma) \subseteq \mathcal{J}(M, \Gamma)$.

We consider actually a more general class of maps than these two: By $\mathcal{C} \mathcal{J}(M, Y)$ (or $\mathcal{C K}(M, Y)$ ) we denote the class of all maps of the form $\varphi \circ \Phi$ for which there is a metric space $\Gamma$ (or an ANR subset $\Gamma$ of a normed space) such that $\varphi: \Gamma \rightarrow Y$ is continuous and $\Phi \in \mathcal{J}(M, \Gamma)$ (or $\Phi \in \mathcal{K}(M, \Gamma)$, respectively).

The classes $\mathcal{C} \mathcal{J}(M, Y)$ and $\mathcal{C K}(M, Y)$ are considerably larger than the classes $\mathcal{J}(M, Y)$ and $\mathcal{K}(M, Y)$. Moreover, in contrast to $\mathcal{J}(M, Y)$, the class $\mathcal{C} \mathcal{J}(M, Y)$ has a nice additivity property:

Proposition 2.7. If $Y$ is a normed space and $S_{1}, S_{2} \in \mathcal{C} \mathcal{J}(M, Y)$ then $S_{1}+S_{2} \in \mathcal{C} \mathcal{J}(M, Y)$. An analogous property holds for $\mathcal{C K}(M, Y)$. 
Proof. By hypothesis, we find for $i=1,2$ metric spaces $\Gamma_{i}$, continuous maps $\varphi_{i}: \Gamma_{i} \rightarrow Y$ and $\Phi_{i} \in \mathcal{J}\left(M, \Gamma_{i}\right)$ (or $\Phi_{i} \in \mathcal{K}\left(M, \Gamma_{i}\right)$ ), respectively, such that $S_{i}=\varphi_{i} \circ \Phi_{i}$. Putting $\Gamma:=\Gamma_{1} \times \Gamma_{2}$, we have the desired representation $S_{1}+S_{2}=$ $\varphi \circ \Phi$ with $\varphi: \Gamma \rightarrow Y, \varphi\left(y_{1}, y_{2}\right):=\varphi_{1}\left(y_{1}\right)+\varphi_{2}\left(y_{2}\right)$, and $\Phi(x):=\Phi_{1}(x) \times \Phi_{2}(x)$. Note that the upper semicontinuity of $\Phi$ follows in view of the compactness of the values $\Phi_{i}(x)$, see [13, Proposition 2.99].

From now on let a maximal monotone map $T: X \multimap X^{*}$ be fixed. We point out that Proposition 2.7 does typically not apply for $S_{1}=T$, since $T$ does often not assume only nonempty compact values. This is why our subsequent degree theory is a proper extension of that from [2].

We use the notation $\stackrel{\circ}{M}$ to denote the interior of a subset $M \subseteq X$.

For an open set $U \subseteq X$, we note that $U_{K}:=U \cap K$ is open in $K$ with relative boundary $\partial_{K} U_{K}:=\bar{U}_{K} \backslash U_{K}$.

We will define a degree $\operatorname{deg}_{K}(T, S, U) \in \mathbb{Z}$ for every open bounded set $U \subseteq$ $X$ and every map $S \in \mathcal{C} \mathcal{J}\left(\bar{U}_{K}, X^{*}\right)$ with ineq ${ }_{\partial_{K} U_{K}}(T+S, K)=\emptyset$ under the following hypotheses:

(1) $\overline{U_{K} \cap V}$ is locally contractible for every finite-dimensional subspace $V \subseteq$ $X$, or $S \in \mathcal{C K}\left(\bar{U}_{K}, X^{*}\right)$.

(2) $S$ belongs to the class $\mathcal{S}^{+}\left(\bar{U}_{K}\right)$.

(3) At least one of the following holds:

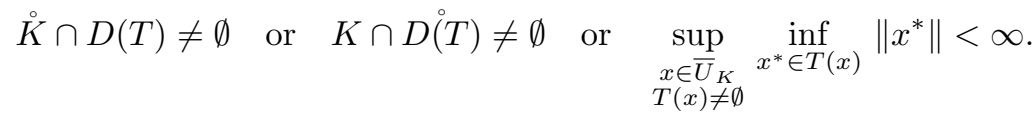

(Actually the class of maps for which the degree is defined will be slightly larger.)

The condition (2.3) may appear very strange, and in fact it is. However, it is unknown to the authors whether it can be dropped: A hypothesis of such a type appears to be crucial for our approach. Note that in the non-inequality case $K=X$ or in case $T=0$ the first or second and third assertion of (2.3) are automatically satisfied, respectively, so that it is not surprising that such a hypothesis (2.3) was never needed in previous literature, since both extensions were never considered simultaneously to our knowledge.

THEOREM 2.8. The degree defined in this paper has the following properties for every open bounded set $U \subseteq X$ satisfying (2.3):

(a) (Homotopy invariance). Let $H \in \mathcal{C} \mathcal{J}\left([0,1] \times \bar{U}, X^{*}\right) \cap \mathcal{S}^{+}([0,1] \times \bar{U})$ be such that $\operatorname{ineq}_{\partial_{K} U_{K}}(T+H(t, \cdot), K)=\emptyset$ for every $t \in[0,1]$. If either $\bar{U}_{K} \cap V$ is locally contractible for every finite-dimensional subspace $V \subseteq$ $X$ or if $H \in \mathcal{C K}\left([0,1] \times \bar{U}, X^{*}\right)$, then $\operatorname{deg}_{K}(T, H(t, \cdot), U)$ is independent of $t \in[0,1]$.

(b) (Excision and Additivity). Let $S \in \mathcal{J}\left(\bar{U}, X^{*}\right) \cap \mathcal{S}^{+}(\bar{U})$, and let $U_{1}, U_{2} \subseteq$ $U$ be open with $U_{1} \cap U_{2}=\emptyset$ and ineq $_{\bar{U}_{K} \backslash\left(U_{1} \cup U_{2}\right)}(T+S, K)=\emptyset$. If 
$\overline{U_{K} \cap V}$ and $\overline{U_{i} \cap K \cap V}(i=1,2)$ are locally contractible for each finitedimensional subspace $V \subseteq X$ or if $S$ assumes only convex values, then

$$
\operatorname{deg}_{K}(T, S, U)=\operatorname{deg}_{K}\left(T, S, U_{1}\right)+\operatorname{deg}_{K}\left(T, S, U_{2}\right) .
$$

(c) (Existence). $\operatorname{deg}_{K}(T, S, U) \neq 0$ implies $\operatorname{ineq}_{U}(T+S, K) \neq \emptyset$.

(d) (Generalized Normalization Property). Let $S \in \mathcal{C} \mathcal{J}\left(\bar{U}_{K}, X^{*}\right) \cap \mathcal{S}^{+}\left(\bar{U}_{K}\right)$ satisfy $\operatorname{ineq}_{\partial_{K} U_{K}}(T+S, K)=\emptyset$. Assume in addition that $\bar{U}_{K} \cap V$ is locally contractible for every finite-dimensional subspace $V \subseteq X$ or that $S \in \mathcal{C K}\left(\bar{U}_{K}, X^{*}\right)$. If there is $x_{0} \in U_{K}$ with $0 \in T\left(x_{0}\right)$ such that

$$
\left\langle x-x_{0}, y\right\rangle \geq 0 \text { for all } x \in \partial_{K} U_{K} \text { and all } y \in S(x),
$$

then $\operatorname{deg}_{K}(T, S, U)=1$.

Let us give a simple application of this degree theory:

Theorem 2.9 (Continuation Principle). Let $U \subseteq X$ be open and bounded and satisfy (2.3). Let $S \in \mathcal{C} \mathcal{J}\left(\bar{U}_{K}, X^{*}\right) \cap \mathcal{S}^{+}\left(\bar{U}_{K}\right)$, and let $C \in \mathcal{C} \mathcal{J}\left([0,1] \times \bar{U}_{K}, X^{*}\right)$ be such that $C\left([0,1] \times \bar{U}_{K}\right)$ is relatively compact. Assume in addition that $\bar{U}_{K} \cap V$ is locally contractible for every finite-dimensional subspace $V \subseteq X$ or that $S \in$ $\mathcal{C K}\left(\bar{U}_{K}, X^{*}\right)$ and $C \in \mathcal{C K}\left([0,1] \times \bar{U}_{K}, X^{*}\right)$. If there is $x_{0} \in U_{K}$ with $0 \in T\left(x_{0}\right)$ such that $\left\langle x-x_{0}, y\right\rangle \geq 0$ for all $x \in \partial_{K} U_{K}$ and $y \in S(x)+C(0, x)$, then at least one of the following alternatives occurs:

(a) There is $t \in[0,1]$ such that ineq $_{\partial_{K} U_{K}}(T+S+C(t, \cdot), K) \neq \emptyset$.

(b) ineq $_{U_{K}}(T+S+C(t, \cdot), K) \neq \emptyset$ for every $t \in[0,1]$.

Proof. By Propositions 2.3 and 2.7, the map $H(t, \cdot):=S+C(t, \cdot)$ belongs to $\mathcal{S}^{+}\left([0,1] \times \bar{U}_{K}\right) \cap \mathcal{C} \mathcal{J}\left([0,1] \times \bar{U}_{K}, X^{*}\right)$ (or to $\mathcal{C K}\left([0,1] \times \bar{U}_{K}, X^{*}\right)$, respectively). Hence, if the first alternative fails, the homotopy invariance implies that $\operatorname{deg}_{K}(T, H(t, \cdot), U)$ is independent of $t$, and by the normalization property, this degree is 1 . Thus, the assertion of the second alternative follows from the existence property.

We use the notation: $B_{r}\left(x_{0}\right)=\left\{x \in X:\left\|x-x_{0}\right\|<r\right\}$.

Corollary 2.10 (Leray-Schauder Alternative). Assume that (2.3) holds with $U_{K}:=K \cap B_{r}(0)$. Let $S \in \mathcal{C} \mathcal{J}\left(\bar{B}_{r}(0), X^{*}\right) \cap \mathcal{S}^{+}\left(\bar{B}_{r}(0)\right)$, and let $C \in$ $\mathcal{C} \mathcal{J}\left(\bar{B}_{r}(0), X^{*}\right)$ be such that $C\left(\bar{B}_{r}(0)\right)$ is relatively compact. If there is $x_{0} \in$ $K \cap B_{r}(0)$ with $0 \in T\left(x_{0}\right)$ such that $\left\langle x-x_{0}, y\right\rangle \geq 0$ for all $x \in K$ with $\|x\|=r$ and all $y \in S(x)$, then at least one of the following alternatives occurs:

(a) There are $t \in[0,1], x \in D(T)$ with $\|x\|=r$, and $y \in(T+S)(x)+t C(x)$ with (1.1).

(b) For every $t \in[0,1]$ there are $x \in D(T) \cap B_{r}(0)$ and $y \in(T+S)(x)+t C(x)$ with (1.1). 
In particular, there are $x \in D(T)$ with $\|x\| \leq r$, and $y \in T(x)+S(x)$ with (1.1); moreover, if this is not the case when $\|x\|=r$, then at least one of the following alternatives occurs:

(c) There are $\lambda>1, x \in D(T)$ with $\|x\|=r$, and $y \in \lambda(T+S)(x)+C(x)$ with (1.1).

(d) There are $x \in D(T)$ with $\|x\| \leq r$, and $y \in(T+S+C)(x)$ with (1.1).

Proof. The first assertion is the special case $U=B_{r}(0), C(t, x)=t C(x)$ of Theorem 2.9, and the second assertion follows from the first with $C=0$ or $\lambda=1 / t$, respectively.

The first specialization $(C=0)$ of Corollary 2.10 can be considered as a variant of a Rothe-type fixed point theorem for variational inequalities with respect to a maximal monotone operator $T$ : Note that in case $K=X$ the assertion means $0 \in T(x)+S(x)$.

The second specialization is an analogue to the Leray-Schauder alternative: Note that concerning $C$ only general topological assumptions (compactness and $\left.C \in \mathcal{C} \mathcal{J}\left(\bar{B}_{r}(0), X^{*}\right)\right)$ are made, and this allows us to obtain assertions concerning inequalities with $T+S$ and $C$. For applications, one can perhaps verify by apriori estimates that all solutions of (1.1) with $y \in(T+S)(x)$ or $y \in \lambda(T+$ $S)(x)+C(x)(\lambda>1)$ have large $\|x\|$ and then can conclude that (for large $r$ ) (1.1) has a solution with $y \in(T+S+C)(x)$.

We point out once more that in case $K=X$, the assertion is a statement about zeroes of maps; for instance, the first case of the alternative means then $0 \in(T+S)(x)+t C(x)$ with $\|x\|=r$ which (for single-valued $C$ ) means in a sense that $t$ is a "nonlinear eigenvalue" of $T+S$ with respect to $C$.

\section{Construction of the degree}

Since $X$ is reflexive and $X^{*}$ is also reflexive and thus weakly compactly generated, there is an equivalent norm on $X$ such that $X$ as well as $X^{*}$ become locally uniformly convex, see e.g. [10, Chapter VII]. Since none of the assertions of Section 2 changes when we pass to an equivalent norm, we may assume this in the following.

However, unless explicitly stated otherwise, we will assume only that $X$ is reflexive and locally uniformly convex and that $X^{*}$ is strictly convex: The reason is that such an equivalent norm is for separable spaces much easier to obtain, see e.g. [9].

A function $f: M \rightarrow Y$ from a metric space $M$ into a Banach space $Y$ is called demicontinuous on $M \subseteq X$ if for any sequence $x_{n} \in M$ with $x_{n} \rightarrow x \in M$ it follows that $f\left(x_{n}\right) \rightarrow f(x)$. Since $M$ is metric and thus first countable this is equivalent to saying that $f: M \rightarrow Y$ is continuous if $Y$ is equipped with the 
weak topology. For the particular case $Y=X^{*}$ note that our hypothesis that $X$ is reflexive implies that the weak and weak* topologies coincide.

Since $X^{*}$ is strictly convex, the duality mapping $J: X \multimap X^{*}$,

$$
J(x):=\left\{x^{*} \in X^{*}:\left\langle x, x^{*}\right\rangle=\left\|x^{*}\right\|\|x\| \text { and }\left\|x^{*}\right\|=\|x\|\right\},
$$

becomes actually single-valued (and $D(J)=X$ by the Hahn-Banach extension theorem); it satisfies

$$
J(\lambda x)=\lambda J(x) \text { for all } \lambda \in \mathbb{R},
$$

and $J: X \rightarrow X^{*}$ is demicontinuous, see e.g. [14, Proposition 32.22]. Since $X^{* *}=$ $X$ is also strictly convex, applying the same observation on $X^{*}$, we obtain that $J^{-1}: X^{*} \multimap X$ is demicontinuous.

REMARK 3.1. A Banach space is said to have the Kadec-Klee property if

$$
\left(x_{n} \rightarrow x \text { and }\left\|x_{n}\right\| \rightarrow\|x\|\right) \Rightarrow\left\|x_{n}-x\right\| \rightarrow 0 .
$$

It follows straightforwardly from the lower semicontinuity of the norm that every locally uniformly convex Banach space has the Kadec-Klee property. Since we assume that $X$ is locally uniformly convex and thus has the Kadec-Klee property and since $J$ and thus $J^{-1}$ are norm-preserving, it follows even that $J^{-1}$ is continuous. If we would assume that $X^{*}$ is also locally uniformly convex (or at least has the Kadec-Klee property) then by the same argument $J$ would be continuous.

Recall that $T$ is maximal monotone if and only if $T$ is monotone and $T+\varepsilon J$ is onto $X^{*}$ for every $\varepsilon>0$. If $T$ is maximal monotone (and since $X$ and $X^{*}$ are strictly convex and reflexive), we can define the Yosida approximations

$$
Y_{\varepsilon}(T):=\left(T^{-1}+\varepsilon J^{-1}\right)^{-1} \text { for all } \varepsilon>0 .
$$

We summarize some results about $Y_{\varepsilon}(T)$ in the following lemma.

Lemma 3.2. $Y_{\varepsilon}(T): X \multimap X^{*}$ is actually single-valued with $D\left(Y_{\varepsilon}(T)\right)=X$, i.e. $Y_{\varepsilon}(T): X \rightarrow X^{*}$. Moreover, for every $x \in X$ the elements $x_{\varepsilon}^{*}=Y_{\varepsilon}(T)(x)$ and

$$
x_{\varepsilon}=X_{\varepsilon}(T)(x):=x-\varepsilon J^{-1}\left(x_{\varepsilon}^{*}\right)
$$

are the unique solutions of the system

$$
x_{\varepsilon}^{*} \in T\left(x_{\varepsilon}\right), \quad J\left(x_{\varepsilon}-x\right)+\varepsilon x_{\varepsilon}^{*}=0 .
$$

$Y_{\varepsilon}(T)$ is monotone, and $Y_{\varepsilon}(T)$ and $X_{\varepsilon}(T)$ are bounded; more precisely,

$$
\begin{aligned}
\bigcup_{\varepsilon \in\left[\varepsilon_{1}, \varepsilon_{2}\right]} Y_{\varepsilon}(T)(B) \cup & \bigcup_{\varepsilon \in\left(0, \varepsilon_{2}\right]} X_{\varepsilon}(T)(B) \\
& \text { is bounded for bounded } B \subseteq X \text { and } 0<\varepsilon_{1} \leq \varepsilon_{2} .
\end{aligned}
$$


We have the estimate

$$
\left\|Y_{\varepsilon}(T)(x)\right\| \leq \inf \left\{\left\|x^{*}\right\|: x^{*} \in T(x)\right\} \quad \text { if } x \in D(T),
$$

and the equalities

(3.6) $Y_{\varepsilon}(T)(x)=Y_{\delta}(T)\left(x+(\delta-\varepsilon) J^{-1}\left(Y_{\varepsilon}(T)(x)\right)\right)$ for all $x \in X$ and $\delta, \varepsilon>0$, and the map $(\varepsilon, x) \mapsto Y_{\varepsilon}(T)(x)$ is demicontinuous. The "resolvent map" $X_{\varepsilon}(T)$ : $X \rightarrow X$ is continuous for every $\varepsilon>0$.

Proof. Since $T^{-1}$ is maximal monotone and $J^{-1}$ is the duality map $X^{*} \multimap$ $X^{* *}=X$, it follows that $T^{-1}+\varepsilon J^{-1}$ is onto $X$, i.e. $D\left(Y_{\varepsilon}(T)\right)=X$. Since, as already observed in [5], system (3.3) has a unique solution $\left(x_{\varepsilon}, x_{\varepsilon}^{*}\right)$ by [6], and since one sees by inserting the definitions of $Y_{\varepsilon}(T)(x)$ and $X_{\varepsilon}(T)(x)$ and using (3.1) that any $x_{\varepsilon}^{*} \in Y_{\varepsilon}(T)(x)$ with corresponding $x_{\varepsilon}=X_{\varepsilon}(T)(x)$ solves (3.3), it follows that $Y_{\varepsilon}(T)(x)$ is single-valued and together with $X_{\varepsilon}(T)(x)$ indeed characterized by (3.3). Now [5, Lemma $1.3(\mathrm{a})]$ shows that $Y_{\varepsilon}(T)$ is a monotone map defined on all of $X$, and thus $X_{\varepsilon}(T)$ is also a map defined on $X^{*}$. From [5, Lemma $\left.1.3(\mathrm{~d})\right]$, we obtain the estimate (3.5). To prove (3.4), we argue as in $[5$, Lemma $1.3(\mathrm{~b})]$ : Fixing $u^{*} \in T(u)$ and multiplying $(3.3)$ by $X_{\varepsilon}(T)(x)-u$ and using the definition of $J$, we obtain that

$$
\begin{aligned}
\varepsilon\left\langle u-X_{\varepsilon}(T)(x), u^{*}\right\rangle & =\left\langle X_{\varepsilon}(T)(x)-u, J\left(X_{\varepsilon}(T)(x)-x\right)\right\rangle \\
& =\left\|X_{\varepsilon}(T)(x)-x\right\|^{2}+\left\langle x-u, J\left(X_{\varepsilon}(T)(x)-x\right)\right\rangle .
\end{aligned}
$$

For $(x, \varepsilon)$ from a bounded set the left-hand side can grow at most affinely with $\left\|X_{\varepsilon}(T)(x)\right\|$ while the right-hand side grows quadratically. This implies that $X_{\varepsilon}(T)(x)$ remains bounded for bounded $x$ and $\varepsilon$. Using (3.3) and that $J$ is norm-preserving, we thus obtain (3.4).

Using (3.3), we have $x-\varepsilon J^{-1}\left(x_{\varepsilon}^{*}\right)=x_{\varepsilon} \in T^{-1}\left(x_{\varepsilon}^{*}\right)$ and hence

$$
x+(\delta-\varepsilon) J^{-1}\left(x_{\varepsilon}^{*}\right) \in T^{-1}\left(x_{\varepsilon}^{*}\right)+\delta J^{-1}\left(x_{\varepsilon}^{*}\right)
$$

for every $\delta>0$. Applying $Y_{\delta}(T)=\left(T^{-1}+\delta J^{-1}\right)^{-1}$ in $(3.2)$ on both sides, we obtain (3.6).

We note further by $[5$, Lemma $1.3(\mathrm{c})]$ that $X_{\varepsilon}(T)$ and $Y_{\varepsilon}(T)$ are demicontinuous. Moreover, since $X$ has the Kadec-Klee property, it follows from $[5$, Remark 1 after Lemma 1.3] that $X_{\varepsilon}(T)$ is even continuous.

The demicontinuity of $(\varepsilon, x) \mapsto Y_{\varepsilon}(T)(x)$ follows by letting $(\varepsilon, x) \rightarrow\left(\delta, x_{0}\right)$ in (3.6), using the uniform boundedness (3.4) and the demicontinuity of $Y_{\delta}(T)$ at $x_{0}$.

REMARK 3.3. If our general assumption that $X$ is locally uniformly convex is replaced by the requirement that $X$ is strictly convex, our proof shows that all assertions of Lemma 3.7 except for the last remain true: The last assertion 
remains true if $X$ has at least the Kadec-Klee property; otherwise we can claim only that $X_{\varepsilon}(T): X \rightarrow X$ is demicontinuous.

REMARK 3.4. If we require that $X$ is reflexive and that both $X$ and $X^{*}$ are strictly convex with the Kadec-Klee property then we can claim even that $(\varepsilon, x) \rightarrow Y_{\varepsilon}(T)(x)$ is norm continuous as a map $(0, \infty) \times X \rightarrow X^{*}$.

Indeed, by Remark 3.3 we still obtain the continuity of $X_{\varepsilon}(T)$, and since $J$ is also continuous by Remark 3.1, we obtain the norm continuity of $Y_{\varepsilon}(T)$ by solving (3.3) for $Y_{\varepsilon}(T)$. As in the proof of Lemma 3.2, this implies the continuity of $(\varepsilon, x) \mapsto Y_{\varepsilon}(T)(x)$ by using (3.4) and (3.6).

REMARK 3.5. In the proof of the (demi)continuity of $(\varepsilon, x) \mapsto Y_{\varepsilon}(T)(x)$, we used the ideas from [1, Lemma 6]. Since this continuity was not known in case $0 \notin T(0)$, the requirement $0 \in T(0)$ was made in the first approaches to degree theory for maximal monotone operators.

The idea of $\left(X_{\varepsilon}(T), Y_{\varepsilon}(T)\right)$ is of course that they approximate $T$ in some graph sense. This is usually made precise by using the following lemma:

Lemma 3.6 (Brezis, Crandall, Pazy). If $y_{n} \in T\left(x_{n}\right), x_{n} \rightarrow x, y_{n} \rightarrow y$, and

$$
\limsup _{n \rightarrow \infty}\left\langle x_{n}-x, y_{n}-y\right\rangle \leq 0
$$

then $x_{n} \rightarrow x, y_{n} \rightarrow y$, and $y \in T(x)$.

Proof. This is [5, Lemma 1.2(1.3)].

Equipped with Lemma 3.6, we can now prove the fundamental step in the construction of our degree.

Lemma 3.7. Let $T: X \multimap X^{*}$ be maximal monotone, $M \subseteq X$ be bounded, and $S \in \mathcal{S}^{+}([0,1] \times M)$ such that ineq $\bar{M}(T+S(t, \cdot), K)=\emptyset$ for every $t \in[0,1]$. Assume that

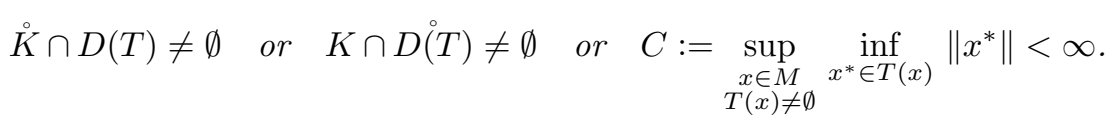

Then there is a positive number $\varepsilon_{0}$ such that

$$
\operatorname{ineq}_{M}\left(Y_{\varepsilon}(T)+S(t, \cdot), K\right)=\emptyset \quad \text { for all } \varepsilon \in\left(0, \varepsilon_{0}\right] \text { and all } t \in[0,1] \text {. }
$$

Proof. Assume by contradiction that there are sequences $\varepsilon_{n}>0$ with $\varepsilon_{n} \rightarrow$ $0, t_{n} \in[0,1]$, and $x_{n} \in \operatorname{ineq}_{M}\left(Y_{\varepsilon_{n}}(T)+S\left(t_{n}, \cdot\right), K\right)$, that is, $x_{n} \in M$, and there are $v_{n} \in S\left(t_{n}, x_{n}\right)$ such that

$$
x_{n} \in K, \quad\left\langle\xi-x_{n}, Y_{\varepsilon_{n}}(T)\left(x_{n}\right)+v_{n}\right\rangle \geq 0 \quad \text { for all } \xi \in K .
$$

Since $M$ and $S([0,1] \times M)$ are bounded and $X$ is reflexive, we can assume without loss of generality that $t_{n} \rightarrow t \in[0,1], x_{n} \rightarrow x \in K$, and $v_{n} \rightarrow v$. Moreover, each of the three hypotheses in (3.7) implies that $u_{n}:=Y_{\varepsilon_{n}}(T)\left(x_{n}\right)$ is bounded. 
Indeed, in the last case $C<\infty$ in (3.7), we have $\left\|u_{n}\right\| \leq \inf \left\{\left\|x^{*}\right\|: x^{*} \in\right.$ $\left.T\left(x_{n}\right)\right\} \leq C$ by $(3.5)$.

In the first case of (3.7), there are $x_{0} \in D(T)$ and $r>0$ such that $B_{r}\left(x_{0}\right) \subseteq$ $K$. Inserting $\xi=x_{0}+y$ with $y \in B_{r}(0)$ into (3.8), we find that

$$
\begin{aligned}
0 \leq & \left\langle x_{0}-x_{n}, Y_{\varepsilon_{n}}(T)\left(x_{n}\right)-Y_{\varepsilon_{n}}(T)\left(x_{0}\right)\right\rangle \\
& +\left\langle x_{0}-x_{n}, Y_{\varepsilon_{n}}(T)\left(x_{0}\right)\right\rangle+\left\langle x_{0}-x_{n}, v_{n}\right\rangle+\left\langle y, u_{n}+v_{n}\right\rangle \\
\leq & \left\|x_{0}-x_{n}\right\|\left(\left\|Y_{\varepsilon_{n}}(T)\left(x_{0}\right)\right\|+\left\|v_{n}\right\|\right)+\left\langle y, u_{n}+v_{n}\right\rangle .
\end{aligned}
$$

Since $v_{n}$ and $x_{n}$ are bounded and $\left\|Y_{\varepsilon_{n}}(T)\left(x_{0}\right)\right\|$ is bounded by (3.5), we obtain that there is some $C_{0} \in[0, \infty)$ such that

$$
\left\langle y, u_{n}+v_{n}\right\rangle \geq-C_{0} \quad \text { for all } n \text { and all } y \in B_{r}(0) .
$$

The latter implies that $\left\|u_{n}\right\| \leq\left\|v_{n}\right\|+C_{0} / r$ is bounded, as claimed.

Finally, in the second case of (3.7), there are $r>0$ and $x_{0} \in K$ with $B_{r}\left(x_{0}\right) \subseteq$ $D(T)$. By [14, Proposition 32.33], we can assume that $T\left(B_{r}\left(x_{0}\right)\right)$ is bounded by some constant $C_{1}$. It follows from $(3.5)$ that $Y_{\varepsilon_{n}}(T)(z)$ is bounded for all $n$ and all $z \in B_{r}\left(x_{0}\right)$ by the same constant $C_{1}$. Since $Y_{\varepsilon_{n}}(T)$ is monotone by Lemma 3.2, we obtain that

$$
\left\langle z-x_{n}, u_{n}\right\rangle \leq\left\langle z-x_{n}, Y_{\varepsilon_{n}}(T)(z)\right\rangle \leq\left\|z-x_{n}\right\| C_{1} \leq C_{2}
$$

is uniformly bounded from above for all $z \in B_{r}\left(x_{0}\right)$ and all $n$. Choosing $\xi=x_{0}$ in (3.8), we find that

$$
0 \leq\left\langle x_{0}-x_{n}, u_{n}+v_{n}\right\rangle \leq\left\langle x_{0}-x_{n}, u_{n}\right\rangle+C_{3},
$$

where $C_{3} \in[0, \infty)$ is independent of $n$. Hence, for every $y \in B_{r}(0)$ we find, putting $z:=x_{0}+y$, that

$$
\left\langle y, u_{n}\right\rangle=\left\langle z-x_{n}, u_{n}\right\rangle+\left\langle x_{n}-x_{0}, u_{n}\right\rangle \leq C_{2}+C_{3} .
$$

Hence, $\left\|u_{n}\right\| \leq\left(C_{2}+C_{3}\right) / r$ is bounded, as claimed.

Thus we can assume that $u_{n} \rightarrow u$. Using (3.8) with $\xi=x$, we find that

$$
0 \geq\left\langle x_{n}-x, u_{n}+v_{n}\right\rangle=\left\langle x_{n}-x, u_{n}\right\rangle+\left\langle x_{n}-x, v_{n}\right\rangle .
$$

We can assume that both summands converge (possibly improperly). If the limit of the last summand is nonpositive then it is zero, because $x_{n} \rightarrow x$ since $S \in \mathcal{S}^{+}([0,1] \times M)$. Hence, the limit of the last summand is nonnegative, and SO

$$
\lim _{n \rightarrow \infty}\left\langle x_{n}-x, u_{n}-u\right\rangle=\lim _{n \rightarrow \infty}\left\langle x_{n}-x, u_{n}\right\rangle \leq 0 .
$$

Putting $\widetilde{x}_{n}:=X_{\varepsilon_{n}}(T)\left(x_{n}\right)=x_{n}-\varepsilon_{n} J^{-1}\left(u_{n}\right)$, we have $u_{n} \in T\left(\widetilde{x}_{n}\right)$ by (3.3). Since $J^{-1}\left(u_{n}\right)$ is bounded, we have $x_{n}-\widetilde{x}_{n} \rightarrow 0$, and so

$$
\lim _{n \rightarrow \infty}\left\langle\widetilde{x}_{n}-x, u_{n}-u\right\rangle \leq 0 .
$$


Since $u_{n} \in T\left(\widetilde{x}_{n}\right)$, this implies with Lemma 3.6 that $u \in T(x), u_{n} \rightarrow u$, and $\widetilde{x}_{n} \rightarrow x$, in particular, $x_{n} \rightarrow x$. From $S \in \mathcal{S}^{+}([0,1] \times M)$, we now obtain that $v \in S(t, x)$. Passing for fixed $\xi \in K$ to the limit in (3.8), we conclude that

$$
\langle\xi-x, u+v\rangle \geq 0 \quad \text { for all } \xi \in K .
$$

Since $x \in \bar{M} \cap K, u \in T(x)$, and $v \in S(t, x)$, we obtain that $x \in \operatorname{ineq}_{\bar{M}}(T+$ $S(t, \cdot), K)$, which is a contradiction to the hypothesis.

In the last part of the previous proof, we used some techniques from the proof of [1, Lemma 9].

Now we come to a rather technical definition. Let $M$ and $\Gamma$ be metric spaces, and $\Phi: M \multimap \Gamma$. Then a single-valued continuous map $f: M \rightarrow \Gamma$ is called an $\varepsilon$-approximation of $\Phi$ if for every $x \in M$ there exists $y \in M$ with $d(x, y)<\varepsilon$ and $z \in \Phi(y)$ with $d(f(x), z)<\varepsilon$. We denote by $\mathcal{A}(M, \Gamma)$ the family of all maps $\Phi: M \multimap \Gamma$ with the following properties:

(1) $\Phi$ is upper semicontinuous with nonempty compact values on $M$.

(2) For every $\varepsilon>0$ there is an $\varepsilon$-approximation of $\Phi$ on $M$.

(3) For every $\delta>0$ there exists $\varepsilon>0$ such that every two $\varepsilon$-approximations $f_{0}, f_{1}: M \rightarrow \Gamma$ are homotopic in the sense that there is a continuous $h:[0,1] \times M \rightarrow \Gamma$ with $h(i, \cdot)=f_{i}(i=0,1)$ such that $h(t, \cdot)$ is a $\delta$ approximation for every $t \in[0,1]$.

According to the remarks in [2], we have the following important examples of maps from $\mathcal{A}(M, \Gamma)$ :

Proposition 3.8. If $\Phi \in \mathcal{J}(M, \Gamma)$ then $\Phi \in \mathcal{A}(M, \Gamma)$ if one of the following holds:

(a) $M$ is an ANR (if $M$ is contained in a finite-dimensional Euclidean space, it is equivalent to require that $M$ is locally contractible [4]), or

(b) $\Gamma$ is a subset of a normed space and $\Phi$ assumes convex values.

Let now $U \subseteq X$ be open and bounded, $U_{K}:=U \cap K$, and $\partial_{K} U_{K}:=\bar{U}_{K} \backslash U_{K}$.

Definition 3.9. $\mathcal{C}\left(U_{K}\right)$ denotes the class of all maps $S=\varphi \circ \Phi$ with the property that there is a metric space $\Gamma$ such that $\varphi: \Gamma \rightarrow X^{*}$ is continuous and $\Phi: X \multimap \Gamma$ is such that $\left.\Phi\right|_{U_{K} \cap V} \in \mathcal{A}\left(\overline{U_{K} \cap V}, \Gamma\right)$ for every finite-dimensional linear subspace $V \subseteq X$.

We define $\mathcal{C}\left([0,1] \times U_{K}\right)$ in the analogous way by requiring $\left.\Phi\right|_{[0,1] \times\left(\overline{U_{K} \cap V}\right)} \in$ $\mathcal{A}\left([0,1] \times\left(\overline{U_{K} \cap V}\right), \Gamma\right)$ for every finite-dimensional linear subspace $V \subseteq X$.

REMARK 3.10. For the special case $\Gamma=\Gamma_{1} \times \Gamma_{2}, \Phi(x)=\Phi_{1}(x) \times \Phi_{2}(x)$, and $\varphi\left(x_{1}, x_{2}\right)=\varphi_{1}\left(x_{1}\right)+\varphi_{2}\left(x_{2}\right)$, the condition $\left.\Phi\right|_{\overline{U_{K} \cap V}} \in \mathcal{A}\left(\overline{U_{K} \cap V}, \Gamma\right)$ is satisfied if

$$
\left.\Phi_{i}\right|_{\overline{U_{K} \cap V}} \in \mathcal{A}\left(\overline{U_{K} \cap V}, \Gamma\right) \quad \text { for } i=1,2 .
$$


However, in the special case $\Gamma_{2}=X^{*}, \varphi_{2}\left(x_{2}\right)=x_{2}$, we relax the definition of $\mathcal{C}\left(U_{K}\right)$ by allowing additionally that condition (3.10) for $i=2$ be replaced by the hypothesis that $\Phi_{2}: X \multimap X^{*}$ is single-valued and demicontinuous on $D\left(\Phi_{2}\right) \supseteq \overline{U_{K} \cap V}$.

This special extension may appear somewhat artificial, but it is necessary to make and was apparently forgotten in [2]. In fact, without such an extension, the argument given in the proof of [2, Proposition 3.29] is faulty, since $J$ is in general only demicontinuous, because $X^{*}$ need not necessarily be locally uniformly convex and thus may fail to have the Kadec-Klee property.

REMARK 3.11. The reader who does not want to extend the definition of $\mathcal{C}\left(U_{K}\right)$ in the technical manner sketched above can instead assume from now on that $X^{*}$ is locally uniformly convex: As remarked earlier, this is no loss of generality, anyway, and by doing so the maps $J$ and $(\varepsilon, x) \mapsto Y_{\varepsilon}(T)(x)$ become even continuous with respect to the norm topology so that the subsequent consideration of demicontinuous functions will actually not be necessary at all.

The main intention of the previous remarks is the validity of the following observation even if $S_{2}$ is only single-valued and demicontinuous on $\bar{U}_{K}$.

Proposition 3.12. If $S_{1}, S_{2} \in \mathcal{C}\left(U_{K}\right)$ then $S_{1}+S_{2} \in \mathcal{C}\left(U_{K}\right)$. An analogous assertion holds for $\mathcal{C K}\left([0,1] \times U_{K}\right)$.

Proof. In view of [2, Theorem 2.2], the same construction as in Proposition 2.7 can be used.

We define $\mathcal{S}_{K}^{+}(U):=\mathcal{S}^{+}\left(\bar{U}_{K}\right) \cap \mathcal{C}\left(U_{K}\right)$ and $\mathcal{S}_{K}^{+}([0,1] \times U):=\mathcal{S}^{+}([0,1] \times$ $\left.\bar{U}_{K}\right) \cap \mathcal{C}\left([0,1] \times U_{K}\right)$.

REMARK 3.13. So far, we never needed in this section that $X$ is separable. In fact, we use that $X$ is separable only in order to use the degree from [2] where this assumption was made. Since we consider this degree from now on, we assume from here that $X$ is separable.

The degree developed in [2] is defined for maps $S \in \mathcal{S}^{+}\left(\bar{U}_{K}\right)$ which satisfy ineq $_{\partial_{K} U_{K}}(S, K)=\emptyset$. (In fact, it is straightforward to check that our technical extension of the class $\mathcal{S}^{+}\left(\bar{U}_{K}\right)$ due to Remark 3.10 does not influence the construction.) It is an integer number which is called $\operatorname{Ind}\left(S, \bar{U}_{K}, K, 0\right)$ in [2]; we prefer to call it a degree,

$$
\operatorname{deg}_{K}(S, U):=\operatorname{Ind}\left(S, \bar{U}_{K}, K, 0\right),
$$

since in case $K=X$ it is related with the zeroes of $S$ (and not with any fixed points which is usually suggested by an index).

In our opinion it is convenient to define the degree for open bounded sets $U \subseteq X$, although in fact it depends only on $U_{K}=U \cap K$, that is, in fact it is 
a relative degree on $K$ : Our attitude corresponds to the fact that in a separable Hilbert space $X$ and if $S=$ id $-A$ with a completely continuous map $A$, this degree corresponds to the Leray-Schauder degree

$$
\operatorname{deg}\left(\mathrm{id}-\left(P_{K} \circ A\right), U, 0\right),
$$

where $P_{K}$ denotes the metric projection onto $K$ (i.e. $P_{K}(x)$ is that element of $K$ with closest distance to $x$ ). Of course, (3.12) is nothing else than the relative fixed point index $\operatorname{ind}_{K}\left(P_{K} \circ A, U_{K}\right)$ so that the notion Ind in (3.11) has some justification. Anyway, to point out the relation with the Browder-Skrypnik degree, we will keep the notion $\operatorname{deg}_{K}$ from (3.11).

The degree $\operatorname{deg}_{K}$ has all the natural properties which one expects from a degree like homotopic invariance (under homotopies $H$ from the class $\mathcal{S}_{K}^{+}([0,1] \times U)$ which satisfy ineq ${ }_{\partial_{K} U_{K}}(H(t, \cdot), K)=\emptyset$ for all $\left.t \in[0,1]\right)$, excision, additivity, and the following normalization property:

Proposition 3.14. If $U \subseteq X$ is open and bounded, $S \in \mathcal{S}_{K}^{+}(U)$ satisfies ineq $_{\partial_{K} U_{K}}(S, K)=\emptyset$, and if there is $x_{0} \in U_{K}$ such that $\left\langle x-x_{0}, y\right\rangle \geq 0$ for all $x \in \partial_{K} U_{K}$ and all $y \in S(x)$ then $\operatorname{deg}_{K}(S, U)=1$.

ProOF. This is shown implicitly in the proof of [2, Theorem 3.23].

Now let a maximal monotone operator $T: X \multimap X^{*}$ be fixed.

Lemma 3.15. If $S \in \mathcal{C}\left(U_{K}\right)$ then $Y_{\varepsilon}(T)+S \in \mathcal{C}\left(U_{K}\right)$ for every $\varepsilon>0$. An analogous result holds for $\mathcal{C}\left([0,1] \times U_{K}\right)$.

Proof. Recall that the map $Y_{\varepsilon}(T)$ is demicontinuous by Lemma 3.2. Hence, the assertion follows from Proposition 3.12 in view of Remark 3.10.

Lemma 3.16. If $S \in \mathcal{S}^{+}(M)$ then $Y_{\varepsilon}(T)+S \in \mathcal{S}^{+}(M)$ for every $\varepsilon>0$. An analogous result holds for $\mathcal{S}^{+}([0,1] \times M)$.

Proof. Lemma 3.2 implies that $Y_{\varepsilon}(T)$ is defined on all of $X$, single-valued, bounded, monotone, and demicontinuous. We obtain from Corollary 2.6 that it is also sequentially demiclosed, and so the assertion follows from Proposition 2.3.

Corollary 3.17. $S \in \mathcal{S}_{K}^{+}(U)$ implies $Y_{\varepsilon}(T)+S \in \mathcal{S}_{K}^{+}(U)$ for every $\varepsilon>0$. An analogous result holds for $\mathcal{S}_{K}^{+}([0,1] \times U)$.

Proof. The assertion is the combination of Lemmas 3.15 and 3.16 with $M=\bar{U}_{K}$

From now on, we assume in addition that $X^{*}$ has the Kadec-Klee property. We recall that this is the case, in particular, if $X^{*}$ is locally uniformly convex, and thus we can assume this without loss of generality. 
We denote by $\mathcal{U}(T, K)$ the family of all bounded open subsets $U \subseteq X$ satisfying (2.3). Given $S \in \mathcal{S}_{K}^{+}(U)$ with $\operatorname{ineq}_{\partial_{K} U_{K}}(T+S, K)=\emptyset$, we define

$$
\operatorname{deg}_{K}(T, S, U):=\lim _{\varepsilon \rightarrow 0^{+}} \operatorname{deg}_{K}\left(Y_{\varepsilon}(T)+S, U\right),
$$

where the degree on the right-hand side denotes the degree (3.11).

Proposition 3.18. The above definition is correct. More precisely, there is $\varepsilon_{0}>0$ such that for all $\varepsilon \in\left(0, \varepsilon_{0}\right]$ the degree $\operatorname{deg}_{K}\left(Y_{\varepsilon}(T)+S, U\right)$ is defined. For every such $\varepsilon_{0}$, the value of this degree is independent of the choice of $\varepsilon$.

Proof. The first assertion follows from Corollary 3.17 and from Lemma 3.7 with $M:=\partial_{K} U_{K}$. For the second assertion, it suffices to show in view of the homotopy invariance of the degree (3.11) that

$$
H(t, x):=Y_{\lambda(t)}(T)(x)+S(x)
$$

belongs to $\mathcal{S}_{K}^{+}([0,1] \times U)$, where $\lambda:[0,1] \rightarrow\left[\varepsilon, \varepsilon_{0}\right]$ is a homeomorphism with $\lambda(1)=\varepsilon_{0}$. Lemma 3.2 implies that $(t, x) \mapsto Y_{\lambda(t)}(T)(x)$ is demicontinuous and by Remark 3.4 even continuous. Hence, Proposition 3.12 implies that $H \in$ $\mathcal{C}\left([0,1] \times U_{K}\right)$.

It remains to show that $H$ belongs to $\mathcal{S}^{+}\left([0,1] \times \bar{U}_{K}\right)$. The boundedness is clear in view of (3.4). However, for the rest of the argument we cannot directly use Proposition 2.3, because we do not know whether $(t, x) \mapsto Y_{\lambda(t)}(T)(x)$ is a jointly monotone family. For this reason, we actually use the (norm) continuity of $Y_{\lambda(\cdot)}(T)(x)$ in the following argument.

Let sequences $t_{n} \in[0,1], x_{n} \in M, y_{n} \in H\left(t_{n}, x_{n}\right)$ be given which satisfy $t_{n} \rightarrow t, x_{n} \rightarrow x, y_{n} \rightarrow y$, and (2.2). We are to show that $y \in H(t, x)$ and $x_{n} \rightarrow x$ for some subsequence (recall Remark 2.2).

We put $u_{n}:=Y_{\lambda\left(t_{n}\right)}(T)\left(x_{n}\right)$ and $v_{n}:=y_{n}-u_{n}$. Then $v_{n} \in S\left(x_{n}\right)$ is bounded, and passing to a subsequence if necessary, we may thus assume that $v_{n} \rightarrow v$. Hence, we have $u_{n} \rightarrow u:=y-v$. The monotonicity of $Y_{\lambda\left(t_{n}\right)}(T)$ implies that

$$
\left\langle x_{n}-x, y_{n}\right\rangle=\left\langle x_{n}-x, u_{n}\right\rangle+\left\langle x_{n}-x, v_{n}\right\rangle \geq\left\langle x_{n}-x, z_{n}\right\rangle+\left\langle x_{n}-x, v_{n}\right\rangle
$$

with $z_{n}:=Y_{\lambda\left(t_{n}\right)}(T)(x)$. Since $X^{*}$ has the Kadec-Klee property, we obtain from Remark 3.4 that $z_{n} \rightarrow z:=Y_{\lambda(t)}(T)(x)$, and so $x_{n} \rightarrow x$ implies that $\left\langle x_{n}-x, z_{n}\right\rangle \rightarrow 0$. Using this in (3.13), we thus obtain from (2.2) that

$$
\limsup _{n \rightarrow \infty}\left\langle x_{n}-x, v_{n}\right\rangle \leq 0 .
$$

Since $S \in \mathcal{S}^{+}\left(\bar{U}_{K}\right)$, we find that $x_{n} \rightarrow x$ and $v \in S(x)$. From Lemma 3.2, we conclude that $u_{n} \rightarrow z$, hence $u=z$, i.e. $y=u+v=z+v \in H(t, x)$, as required. Thus, we have shown that $H \in \mathcal{S}^{+}\left([0,1] \times \bar{U}_{K}\right)$. Consequently, $H$ belongs to $\mathcal{S}_{K}^{+}([0,1] \times U)$. 
THEOREM 3.19. The degree defined above has the following properties for every open bounded set $U \subseteq X$ satisfying (2.3):

(a) (Homotopy invariance). If $H \in \mathcal{S}_{K}^{+}([0,1] \times U)$ is such that ineq ${ }_{\partial_{K} U_{K}}(T+$ $H(t, \cdot), K)=\emptyset$ for all $t \in[0,1]$, then

$$
\operatorname{deg}_{K}(T, H(t, \cdot), U) \quad \text { is independent of } t \in[0,1] .
$$

(b) (Excision and Additivity). If $S \in \mathcal{S}_{K}^{+}(U)$ and $U_{1}, U_{2} \subseteq U$ are open with $U_{1} \cap U_{2}=\emptyset$ and ineq $_{\bar{U} \backslash\left(U_{1} \cup U_{2}\right)}(T+S, K)=\emptyset$, then

$$
\operatorname{deg}_{K}(T, S, U)=\operatorname{deg}_{K}\left(T, S, U_{1}\right)+\operatorname{deg}_{K}\left(T, S, U_{2}\right) .
$$

(c) (Existence). $\operatorname{deg}_{K}(T, S, U) \neq 0$ implies $\operatorname{ineq}_{U}(T+S, K) \neq \emptyset$.

(d) (Generalized Normalization Property). Let $S \in \mathcal{S}_{K}^{+}(U)$ satisfy

$$
\text { ineq }_{\partial_{K} U_{K}}(T+S, K)=\emptyset .
$$

If there is $x_{0} \in U_{K}$ with $0 \in T\left(x_{0}\right)$ such that (2.4) holds, then

$$
\operatorname{deg}_{K}(T, S, U)=1
$$

Proof. The homotopy invariance follows directly from the definition and from the homotopy invariance of the degree (3.11), since by Lemma 3.7 the number $\varepsilon_{0}$ in the definition of $\operatorname{deg}_{K}(T, H(t, \cdot), U)$ can be chosen independently of $t$.

Similarly, applying Lemma 3.7 with the closed set $M:=\overline{U_{K} \backslash\left(U_{1} \cup U_{2}\right)}$, we can choose $\varepsilon_{0}>0$ so small that $\operatorname{ineq}_{M}\left(Y_{\varepsilon}(T)+S, K\right)=\emptyset$ for all $\varepsilon \in\left(0, \varepsilon_{0}\right]$, and so the excision/additivity follows from the corresponding property of the degree (3.11).

The existence property is the special case $U_{1}=U_{2}=\emptyset$ of the excision/additivity.

In order to prove the normalization property, it suffices to show that

$$
\operatorname{deg}_{K}\left(Y_{\varepsilon}(T)+S, U\right)=1 \text { for all } \varepsilon>0
$$

satisfying ineq ${ }_{\partial_{K} U_{K}}\left(Y_{\varepsilon}(T)+S, K\right)=\emptyset$ (by Proposition 3.18 we know that the latter holds at least for small $\varepsilon>0$ ). To see this, we use Proposition 3.14 with $\widetilde{S}:=Y_{\varepsilon}(T)+S$. Hence, we are to show that for every $x \in \partial_{K} U_{K}$ the inequalities

$$
\left\langle x-x_{0}, y\right\rangle \geq 0 \text { for all } y \in \widetilde{S}(x)
$$

hold. In view of (2.4), it suffices to prove

$$
\left\langle x-x_{0}, Y_{\varepsilon}(T)(x)\right\rangle \geq 0 .
$$

To see (3.14), we note that in view of $0 \in T\left(x_{0}\right)$ system (3.3) has the solution $\left(x_{\varepsilon}, x_{\varepsilon}^{*}\right)=\left(x_{0}, 0\right)$, and so Lemma 3.2 implies $Y_{\varepsilon}(T)\left(x_{0}\right)=0$. Now (3.14) follows from the monotonicity of $Y_{\varepsilon}(T)$ (Lemma 3.2). 
In view of Proposition 3.8, the degree described in Section 2 is a special case of our degree, and Theorem 2.8 is a special case of Theorem 3.19.

\section{REFERENCES}

[1] G.M. Asfaw and A.G. Kartsatos, A Browder topological degree theory for multi-valued pseudomonotone perturbations of maximal monotone operators, Adv. Math. Sci. Appl. 22 (2012), 91-148.

[2] I. Benedetti And V.V. Obukhovskil, On the index of solvability for variational inequalities in Banach spaces, Set-Valued Anal. 16 (2008), 67-92.

[3] I. Benedetti And P. Zecca, Relative topological degree and variational inequalities, Mediter. J. Math. 3 (2006), 47-65.

[4] K. Borsuk, Theory of Retracts, Polish Scientific Publ., Warszawa, 1967.

[5] H. Brézis, M. G. Crandall and A. Pazy, Perturbations of nonlinear maximal monotone sets in Banach space, Comm. Pure Appl. Math. 23 (1970), 123-144.

[6] F.E. Browder, Nonlinear maximal monotone mappings in Banach spaces, Math. Ann. 175 (1968), 81-113.

[7] _ Nonlinear operators and nonlinear equations of evolution in Banach spaces, Proc. Sympos. Pure Math., vol. XVIII, Part 2, Amer. Math. Soc., Providence, R.I., 1976.

[8] _ Fixed point theory and nonlinear problems, Bull. Amer. Math. Soc. 9 (1983), $1-39$.

[9] M.M. DAY, Normed Linear Spaces, 3rd ed., Springer-Verlag, 1973.

[10] R. Deville, G. Godefroy And V. Zizler, Smoothness and Renormings in Banach Spaces, Longman, 1999.

[11] B.-T. Kien, M.-M. Wong And N.-C. Wong, On the degree theory for general mappings of monotone type, J. Math. Anal. Appl. 340 (2008), 707-720.

[12] I.V. Skrypnik, Nonlinear Elliptic Boundary Value Problems, Teubner-Verlag, 1986.

[13] M. V̈̈тн, Topological Analysis. From the Basics to the Triple Degree for Nonlinear Fredholm Inclusions, de Gruyter-Verlag, 2012.

[14] E. Zeidler, Nonlinear Functional Analysis and its Applications, vol. II/B, SpringerVerlag, 1990.

[15] S.-S. Zhang AND Y.-Q. Chen, Degree theory for multivalued $(S)$-type mappings and fixed point theorems, Appl. Math. Mech. 11 (1990), 441-454.

IN-SoOK KIM

Department of Mathematics

Sungkyunkwan University

Suwon 440-746, REPUBLIC OF KOREA

E-mail address: iskim@skku.edu

MARTIN VÄTH

Freie Universität Berlin

Fachbereich Mathematik und Informatik (WE1)

Arnimallee 3

D-14195 Berlin, GERMANY

E-mail address: vaeth@mathematik.uni-wuerzburg.de

TMNA : VOLUME $47-2016-\mathrm{N}^{\mathrm{O}} 2$ 